\title{
Welches ist der Weisheit letzter Schluss?
}

Fragestellung: Sowohl Lamotrigin (LTG) als auch Levetiracetam (LEV) gehören zu den moderneren Antikonvulsiva (AED), die gern auch schon zu Therapiebeginn eingesetzt werden. Diese Studie vergleicht beide Medikamente bezüglich Wirksamkeit und Verträglichkeit in der initialen Monotherapie bei fokalen, generalisierten und unklassifizierten Epilepsien.

Hintergrund: Gerade zu Therapiebeginn ist die Wahl des richtigen AED entscheidend für den weiteren Verlauf der Epilepsie bezüglich Anfallsfreiheit, Verträglichkeit und Compliance. Dies ist nicht immer leicht, denn in mehr als $20 \%$ der Fälle wissen wir nach dem ersten Anfall nicht, welches Epilepsiesyndrom sich dahinter verbirgt. Dies bedingt den Einsatz eines potenziell breit wirksamen AED. Die SANAD-Studie hatte 2007 LTG als Mittel der Wahl herausarbeiten können, es erfolgte jedoch kein Vergleich mit LEV. Letzteres ist allerdings aufgrund seiner Pharmakokinetik mit rasch erreichbaren suffizienten Wirkspiegeln und seiner guten Kombinierbarkeit bei geringer Interaktion ein sehr beliebtes Medikament geworden.

Patienten und Methodik: Primärer Endpunkt der randomisierten, kontrollierten Open-label-Multicenterstudie war Anfallsfreiheit in den ersten sechs Therapiewochen. Die Behandlungsperiode betrug insgesamt 26 Wochen. Daneben wurden die Verträglichkeit der AED und die Lebensqualität der Patienten in den Behandlungsarmen verglichen. Insgesamt wurden 409 Patienten über zwölf Jahre mit einer neu diagnostizierten fokalen oder generalisierten Epilepsie eingeschlossen oder Patienten die einen oder mehrere unprovozierte Anfälle hatten mit zu erwartendem hohen Wiederholungsrisiko. Aufdosiert wurde LEV bis 2.000 $\mathrm{mg} / \mathrm{d}$, LTG bis $200 \mathrm{mg} / \mathrm{d}$. Die initiale Dosis betrug bei LEV 500 mg, bei LTG $25 \mathrm{mg}$, die Dosissteigerungen erfolgten wöchentlich beziehungsweise bei LTG zweiwöchentlich in den jeweils üblichen und zugelassenen Steigerungsschritten. Die Zieldosis sollte somit nach 22 beziehungsweise 71 Tagen erreicht sein. Danach waren weitere Dosisveränderungen in 500- beziehungsweise 50-mgSchritten erlaubt, je nach Verträglichkeit und Anfallssituation.

Ergebnisse: Sechs Wochen nach der Randomisierung waren 67,5\% der mit LEV und 64\% der mit LTG behandelten Patienten anfallsfrei. Während der gesamten Therapiezeit betrug die Anfallsfreiheit unter LEV 45,2\%, unter LTG 47,8\%. Nebenwir-

Rosenow F, Schade-Brittinger C, Burchardi N et al; for the LaLiMo Study Group. The LaLiMo trial: lamotrigine compared with levetiracetam in the initial 26 weeks of monotherapy for focal and generalized epilepsy - an open-label, prospective, randomised controlled multicenter study. J Neurol Neurosurg Psychiatry 2012; 83: $1093-8$ kungen, die zum Studienabbruch führten, traten bei 17/204 unter LEV und bei 8/201 unter LTG auf, ansonsten waren Nebenwirkungen prozentual gleich verteilt.

Schlussfolgerungen: LEV und LTG sind bei neu diagnostizierten Epilepsien gleich wirksam und gleich verträglich.

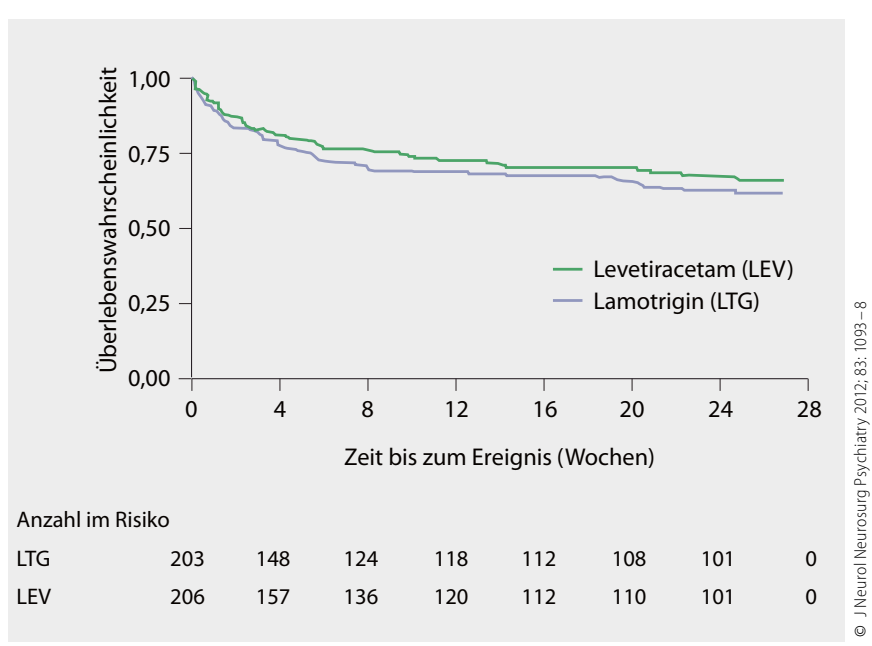

1 Anfallsfreie Zeit unter Lamotrigin und Levetiracetam

\section{- Kommentar von Vivien Homberg, Bad Berka}

\section{Alles bleibt beim Alten}

Das Ergebnis erstaunt ein wenig, vor allem wenn man bisher so viel Ungeduld beim mühsamen Auftitrieren von LTG empfunden hat. Am Ende ist es aber doch das mindestens gleich potente Medikament, und, ehrlich gesagt, man hätte LEV aufgrund seines sportlichen Einsatzes mehr zugetraut. Wider erwarten scheint das schnelle Aufdosieren nichts bezüglich potenzieller Anfallsfreiheit zu bringen, rascher erreichte hohe Wirkspiegel schützen also nicht zwangsläufig vor Anfallsrezidiven, eher erkauft man sich damit Nebenwirkungen wie Müdigkeit (32,8\% unter LEV vs. 16,4\% unter LTG) und die bekannten psychiatrischen Nebenwirkungen. Was spricht also dagegen LTG weiterhin first-line einzusetzen? Bislang nichts. Auch diese Studie unterstreicht nochmals die ausgesprochen gute Wirksamkeit dieses viel erprobten Medikaments. Bei initial hoher Anfallsaktivität ist jedoch auch in Zukunft LEV aufgrund des schnell zu erreichenden Wirkspiegels und der zur Verfügung stehenden i.v.-Applikationsmöglichkeit sicherlich ein unschlagbarer Kandidat um rasch Anfallsreduktion zu erreichen.

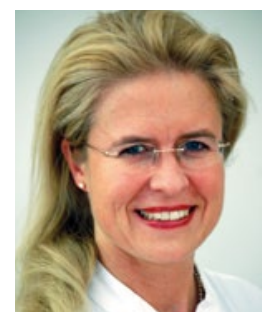

Dr. med. Vivien Homberg, Bad Berka

Chefärztin der Klinik für Neurologie, Zentralklinikum Bad Berka E-Mail:Vivien.Homberg@zentralklinik.de 